\title{
Reconhecimento Computadorizado de Padrões Inflamatórios de Sacroiliíte em Imagens de Ressonância Magnética
}

\author{
Matheus Calil Faleiros ${ }^{1,2}$, José Raniery Ferreira Junior ${ }^{1,2}$, Eddy Javala Jens ${ }^{1}$, Vitor Faeda Dalto ${ }^{1}$, \\ Marcello Henrique Nogueira-Barbosa ${ }^{1}$, Paulo Mazzoncini de Azevedo-Marques ${ }^{1}$ \\ ${ }^{1}$ Centro de Ciências das Imagens e Física Médica - Faculdade de Medicina de Ribeirão Preto \\ Universidade de São Paulo (USP) - Ribeirão Preto - SP - Brasil \\ 2 Programa de Pós-Graduação Interunidades em Bioengenharia - Universdade de São Paulo (USP) - São \\ Carlos - SP - Brasil \\ \{matheus.faleiros, jose.raniery, eddy.zavala, vitor.dalto\}@usp.br, \{marcello, pmarques\}@fmrp.usp.br
}

\begin{abstract}
The reference standard to evaluate active inflammation of sacroiliac joints (SJ) in spondyloarthritis is magnetic resonance imaging (MRI). However, it may be challenging to specialists due to clinical variability. In this context, we aim to recognize inflammatory patterns of SJ using gray-level, texture, and spectral features. Features have been extracted from MRI exams of 51 patients and selected by the ReliefF feature selection method. Image classification was performed by machine learning methods and was assessed by the area under the receiver operating characteristic curve, with a 10-fold cross validation. Results have shown that the five nearest neighbors classifier presented the best performance for inflammatory pattern recognition.
\end{abstract}

Resumo. O método referência para avaliar a inflamação nas articulações sacroilíacas (AS) em espondiloartrites é a ressonância magnética (RM). Porém, ele pode apresentar desafios para especialistas devido a sua variabilidade clínica. Neste contexto, este trabalho visa reconhecer padrões inflamatórios de AS em imagens de RM utilizando atributos de níveis de cinza, textura e espectrais. Os atributos foram extraídos de 51 pacientes e selecionados pelo método ReliefF. A classificação foi realizada por métodos de aprendizado de máquina e avaliados pela área sob a curva ROC utilizando validação cruzada 10-fold. Resultados mostraram que o método de cinco vizinhos próximos apresentou maior precisão do que os outros classificadores.

\section{Introdução}

Espondiloartrites (EpA) são um grupo de doenças inflamatórias crônicas que apresentam características clínicas e radiológicas comuns. As EpA causam comprometimento do esqueleto apendicular, coluna vertebral e articulações sacroilíacas (AS) [Barros 2011].

O método mais comum para diagnóstico precoce desta doença é a ressonância magnética (RM), pois ela é capaz de detectar o edema ósseo nas AS. Contudo, a interpretação visual das imagens de RM com EpA pode ter variação significativa entre observadores distintos e elevado grau de dificuldade na rotina clínica [Pialat 2016].

Considerando esse contexto, o objetivo do trabalho aqui apresentado é realizar o reconhecimento computadorizado de padrões inflamatórios de AS em imagens de RM, para auxiliar o diagnóstico precoce das EpA. 


\section{Material e Métodos}

\subsection{Aquisição e Processamento das Imagens}

O trabalho foi aprovado pelo Comitê de Ética em Pesquisa da Instituição. Foram selecionados retrospectivamente exames de RM de 51 pacientes com diagnóstico de espondiloartrites. As imagens analisadas foram adquiridas no plano coronal na sequência ponderação T2-SPAIR (spectral attenuated inversion recovery) [Dalto 2017]. Para cada exame foram obtidas 6 imagens (cortes) consecutivas da região das articulações sacroilíacas no plano coronal, que foram avaliadas de acordo com os critérios de diagnóstico de RM da Spondyloarthrites Research Consortium of Canada (SPARCC) [Maksymowych 2005]. As articulações esquerda e direita de cada imagem foram identificadas e manualmente segmentadas por um radiologista. As regiões contendo as articulações foram inseridas em um fundo preto para, em seguida, passarem por um algoritmo de processamento de imagens chamado warp, que faz uma transformação geométrica para que a região de interesse ocupe toda imagem (Figura 1). Aplicou-se também um algoritmo de Transformada de Fourier nas imagens pós warp, para possibilitar a extração de atributos espectrais.
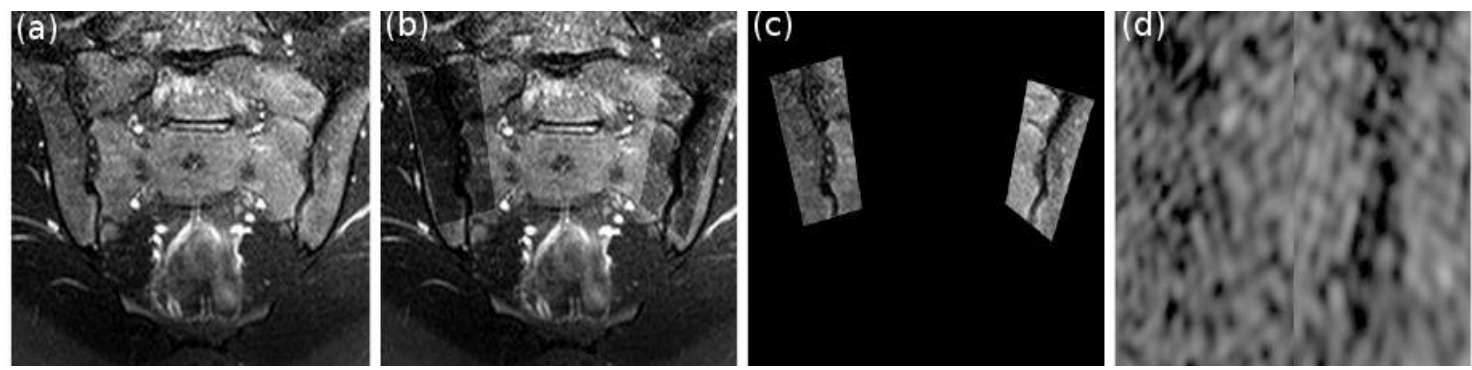

Figura 1. Processamento das imagens de RM. (a) Imagem de RM obtida; (b) Articulações identificadas por um radiologista; (c) Articulações segmentadas em fundo preto; (d) Articulações processadas pelo warp

\subsection{Extração e Seleção dos Atributos}

De cada uma das imagens processadas pelo método warp foram extraídos 7 atributos de níveis de cinza: Média, Desvio Padrão, Assimetria, Kurtosis, Variância, Coeficiente de Desvio e Valor Máximo de nível de cinza, 14 atributos extraídos da matriz de coocorrência e propostos por [Haralick 1973], e 18 extraídos do histograma propostos por [Tamura 1978]. Os atributos de [Haralick 1973] foram Segundo Momento Angular, Contraste, Correlação, Variância, Momento da Diferença Inversa, Média da Soma, Entropia da Soma, Variância da Soma, Entropia, Variância da Diferença, Entropia da Diferença, duas Medidas de Informação de Correlação e Coeficiente de Correlação Máxima. Já para [Tamura 1978] foram Contraste, Granularidade e Direcionalidade, obtidos em 16 direções. Os algoritmos de extração de atributos foram implementados em Java, utilizando-se a biblioteca open-source [JFeatureLib].

Do espectro de Fourier de cada imagem, em coordenadas polares, foram extraídos 39 atributos, sendo eles: Maior valor para cada valor de raio (10 raios), Maior valor para cada valor do ângulo Theta em cada um dos raios (18 Thetas), Energia Cumulativa para cada raio e Integral de metade da função de Fourier [Gonzalez e Woods 1993]. Os atributos de Fourier foram implementados na linguagem de programação Java, utilizando a transformada de Fourier disponível no software opensource ImageJ [Schneider 2012]. 
O vetor de atributos de cada paciente (exame) foi construído a partir da média e do desvio padrão de cada um dos atributos extraídos das suas 6 imagens, resultando em um vetor de 154 dimensões para cada exame.

A seleção de atributos mais relevantes foi realizada pelo método ReliefF [Kononenko 1994], com o método de busca do tipo Ranker, implementado pelo software open-source Weka [Frank 2016]. O ReliefF avalia o atributo individualmente de acordo com o seu valor para a classe majoritaria entre múltiplas instâncias mais próximas do conjunto de dados fornecidos [Kononenko 1994]. Com a busca do tipo Ranker, os atributos são listados em ordem decrescente de acordo com a relevância atribuída pelo ReliefF.

\subsection{Classificação das Imagens}

Para a classificação, foram utilizados os métodos clássicos de aprendizado de máquina K Vizinhos Mais Próximos (valores de K de 1 a 5), rede neural artificial Perceptron Multicamadas, Naive Bayes, Floresta Aleatória e Árvore de Decisão J48. Os classificadores encontram-se implementados na ferramenta Weka [Frank 2016].

A combinação dos atributos com os classificadores utilizados foi feita de forma exaustiva, ou seja, os $\mathrm{N}$ primeiros atributos dados pelo ReliefF foram utilizados por todos os classificadores, para $155>\mathrm{N}>0$.

\section{Resultados}

Para avaliar cada combinação de atributos para cada classificador, foi calculada a área sob a curva ROC (AUC - area under the Receiver Operating Characteristic curve) utilizando o método de validação cruzada 10-fold.

A Tabela 1 mostra uma relação dos maiores valores de AUC para cada classificador e a respectiva dimensão do vetor de atributos obtido pelo método ReliefF. Os valores de K são referentes ao classificador K Vizinhos Mais Próximos.

Tabela 1. Resultados do reconhecimento de padrões inflamatórios

\begin{tabular}{|c|c|c|}
\hline Classificador & AUC & Número de Atributos \\
\hline Naive Bayes & 0.90 & 19 \\
\hline Perceptron Multicamadas & 0.95 & 13 \\
\hline $\mathrm{K}=1$ & 0.90 & 5 \\
\hline $\mathrm{K}=2$ & 0.94 & 5 \\
\hline $\mathrm{K}=3$ & 0.95 & 19 \\
\hline $\mathrm{K}=4$ & 0.95 & 19 \\
\hline $\mathrm{K}=5$ & 0.96 & 17 \\
\hline Árvore de Decisão J48 & 0.76 & 5 \\
\hline Floresta Aleatória & 0.93 & 45 \\
\hline
\end{tabular}

\section{Conclusão}

Esse trabalho apresentou os resultados iniciais de uma pesquisa voltada para o reconhecimento computadorizado de padrões inflamatórios de sacroileíte em imagens 
de ressonância magnética. O uso dos valores de média e desvio padrão dos atributos extraídos de cada corte das imagens dos exames avaliados, em conjunto com os métodos de seleção de atributos e classificadores testados, mostrou-se uma abordagem promissora para o reconhecimento dos padrões indicativos de atividade inflamatória da doença, com destaque para o classificador do tipo KNN com K igual a cinco.

\section{Referências}

Barros, P. (2011) "Epidemiology of Spondyloarthritis in Brazil”, The American Journal of the Medical Sciences, 341(4), 287-288.

Dalto, V., Assad, R., Crema, M., Louzada-Junior, P., Nogueira-Barbosa, M. (2017) "MRI assessment of bone marrow oedema in the sacroiliac joints of patients with spondyloarthritis: is the SPAIR T2w technique comparable to STIR?”, European Radiology, doi:10.1007/s00330-017-4746-7.

Frank, E., Hall, M., Witten, I. (2016) “The WEKA Workbench”. Online Appendix for Data Mining: Practical Machine Learning Tools and Techniques, Fourth Edition.

Gonzalez, R., Woods, R. (1993) Digital image processing, Addison-Wesley.

Haralick, R., Shanmugam, K., Dinstein, I. (1973) "Textural Features for Image Classification”. IEEE Transactions on Systems, Man, and Cybernetics, 3(6), 610621.

JFeatureLib open source project. http://github.com/locked-fg/JFeatureLib, 02/05/2016. Fontes: Haralick.java - Autor: graf ; Tamura.java - Autor: Marko Keuschnig \& Christian Penz ; Histogram.java - Autor: graf;

Kononenko, I. (1994) "Estimating attributes: analysis and extensions of RELIEF", Machine Learning: ECML-94. Springer Berlin Heidelberg, 171-182.

Maksymowych, W., Inman, R., Salonen, D., Dhillon, S., Williams, M., Stone, M., Spady, B., Palsat, J., Lambert, R. (2005) "Spondyloarthritis research Consortium of Canada magnetic resonance imaging index for assessment of sacroiliac joint inflammation in ankylosing spondylitis”, Arthritis \& Rheumatism, 53(5), 703-709.

Pialat, J., Di Marco, L., Feydy, A., Peyron, C., Porta, B., Himpens, P., Boudrigua, A., Aubry, S. (2016) “Sacroiliac joints imaging in axial spondyloarthritis”, Diagnostic and Interventional Imaging, 97(7), 697-708.

Schneider, C., Rasband, W., Eliceiri, K. (2012) "NIH Image to ImageJ: 25 years of image analysis", Nature Methods 9(7): 671-675.

Tamura, H., Mori, S., Yamawaki, T. (1978) “Textural Features Corresponding to Visual Perception”. IEEE Transactions on Systems, Man, and Cybernetics, 8(6), 460-473. 